\title{
Real time fixation point monitoring system for photocoagulation of juxtafoveal neovascularisation
}

\author{
Seiyo Harino, Yoshiko Iwahashi, Yusuke Oshima, Miki Sawa, Hirokazu Nakamura
}

\begin{abstract}
Backgroundlaims-A new real time monitoring system has been developed to locate the fixation point during juxtafoveal laser photocoagulation.

Method-The red diode laser beam is combined coaxially with the illumination beam to image a cross in the focal plane of the slit lamp, which allows projection of a red cross onto the patient's fundus. 27 patients with juxtafoveal choroidal neovascularisation were treated by photocoagulation using this system.

Results-13 (48\%) patients whose visual acuity ranged from $20 / 200$ to $20 / 40$ answered that it was easier to keep the focus on the cross target image than on the aiming beam. The patient maintained stable fixation throughout the treatment. The laser treatment was completed without foveal damage near the fixation point in all patients.

Conclusion-The real time fixation monitoring system should allow surgeons to treat juxtafoveal lesions with laser photocoagulation more safely and accurately.

(Br F Ophthalmol 2000;84:1053-1055)
\end{abstract}

Laser photocoagulation is a beneficial treatment for patients with juxtafoveal choroidal neovascularisation (CNV) resulting from age related macular degeneration. ${ }^{1}$ To treat juxtafoveal neovascularisation, laser burns should be applied to the entire CNV lesion and extend $100 \mu \mathrm{m}$ beyond the lesion when the posterior boundary of the CNV is more than $100 \mu \mathrm{m}$

Department of Ophthalmology, Yodogawa Christian

Hospital, Osaka, Japan

$S$ Harino

Y Iwahashi

M Sawa

Department of

Ophthalmology, Osaka

University Medical

School, Suita-City,

Japan

Y Oshima

Nidek Co, Ltd, Gamagori, Japan H Nakamura

Correspondence to: Dr Harino, Department of Ophthalmology, Yodogawa Christian Hospital, 2-9-26 Awaji, Higashiyodogawa-ku, Osaka 533-0032, Japan sharino@jb3.so-net.ne.jp

Accepted for publication 13 April 2000 from the foveal centre. ${ }^{2}$ Although treatment close to the foveal centre provides the patient

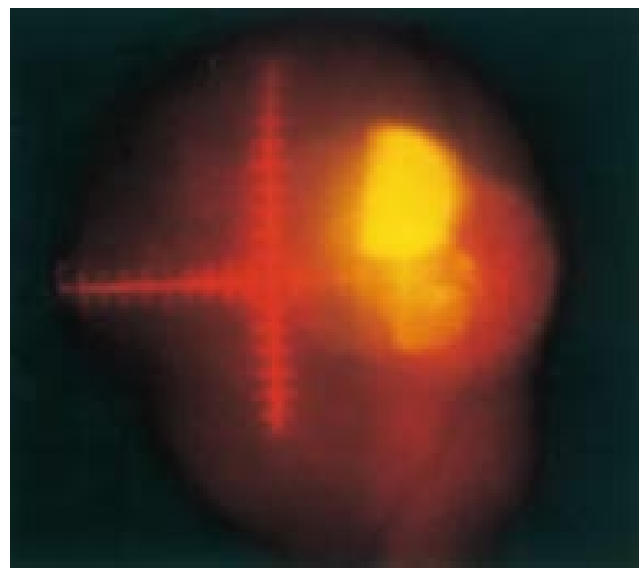

Figure 1 A real image of the cross target formed by the diode laser beam $(633 \mathrm{~nm})$ on a normal fundus. This was photographed in $35 \mathrm{~mm}$ film for a presentation. The brightest white spots are reflections of the contact lens.

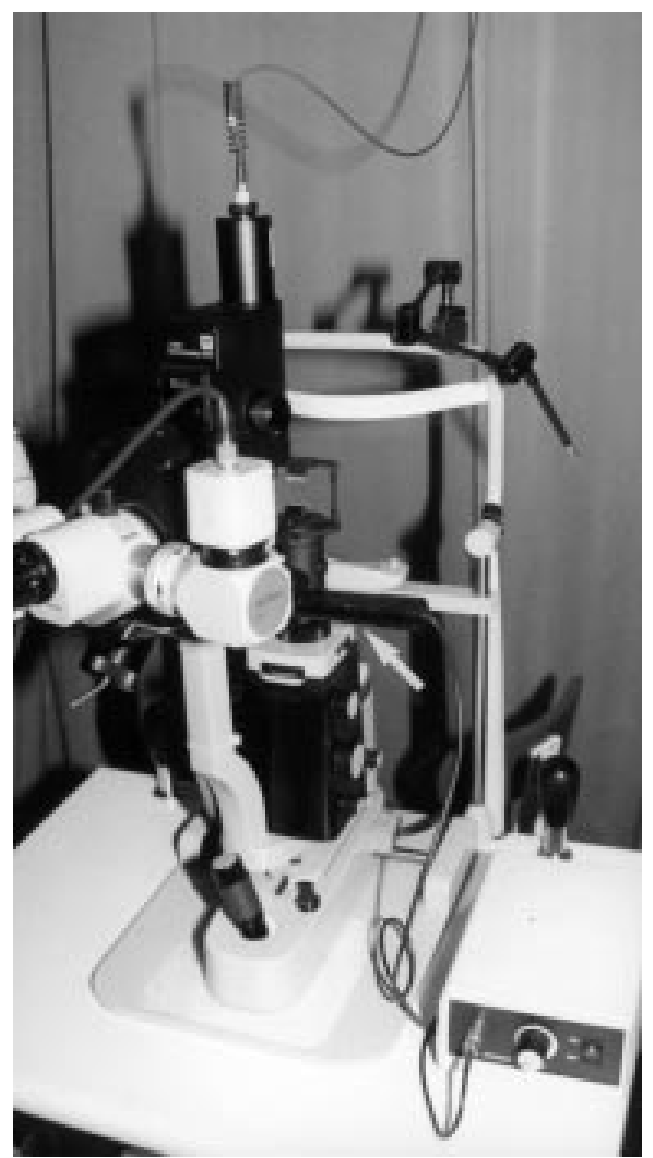

Figure 2 Photograph of the apparatus attached to the slit lamp (Zeiss 30 SL) in an argon/dye laser instrument, which projects a cross onto the fundus (arrow). There is a diode laser source which produces the cross target image on the table.

with the best chance of avoiding further severe visual loss, ${ }^{2}$ inadvertent foveal ablation is a complication. To avoid iatrogenic foveolar damage, the exact retinal fixation point must be determined during laser photocoagulation. We designed a new laser treatment apparatus which can determine the exact point continuously during treatment as the patient fixates on the centre of a cross projected onto the fundus.

Materials and methods

The red cross (Fig 1) $(4 \times 4 \mathrm{~mm}$ in length, 0.05 $\mathrm{mm}$ in thickness, $0.2 \mathrm{~mm}$ between points on the scale) is projected onto the fundus through an apparatus simply attached to the split mirror head of the illumination column of the slit lamp (Zeiss $30 \mathrm{SL}$ in a Nidek ADC-8000S argon/dye laser) (Fig 2). The schematic optical pathway is shown in Figure 3. The red diode laser beam $(633 \mathrm{~nm})$ is combined coaxially with the illumination beam to form a cross in 


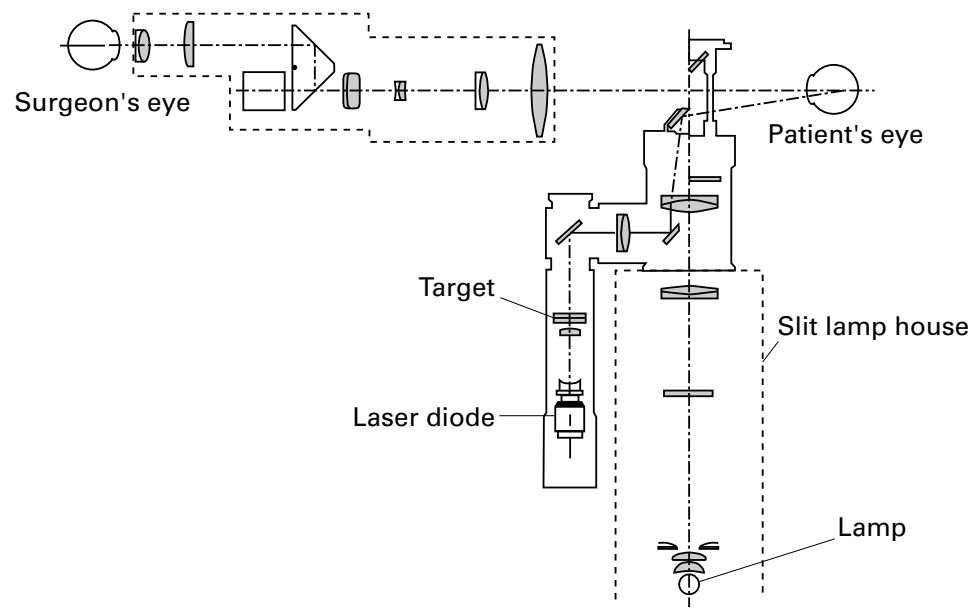

Figure 3 The optical diagram in the apparatus attached to the Zeiss slit lamp.

the focal plane of the slit lamp. Thus, the cross image is independent of the laser aiming beam. We can adjust the distance between the centre of the target and the laser treatment beam. Images are usually monitored and recorded on an S-VHS video recorder (Sony, SVO-260) through the slit lamp microscope (Fig 5). A real image of the cross target on a normal fundus was photographed in $35 \mathrm{~mm}$ film for demonstration in Figure 1.

We have used this system on 27 patients with juxtafoveal choroidal neovascularisation. The posterior edge of the neovascularisation was within $199 \mu \mathrm{m}$ from the fixation point. $^{2}$ Patients' corrected visual acuity ranged from $20 / 200$ to $20 / 40$. Patients were asked which is easier for fixation - the centre of the cross target image or the original laser aiming beam (wavelength is $630 \mathrm{~nm}$ ). The surgeon monitored the stability of the fixation throughout the laser procedure.

\section{Case report}

A 45 year old woman underwent treatment for juxtafoveal CNV with our system. Her corrected visual acuity was $18 / 20$ in the left eye at the initial examination. Ophthalmoscopy and fluorescein angiography (Fig 4) revealed juxtafoveal CNV in the left myopic fundus (refractive error -8.5 dioptres). Her vision decreased to $20 / 40$ during the following month because the CNV enlarged. After a full explanation of the advantages and disadvantages of the laser treatment, including foveal damage or late scar expansion, the patient chose to undergo treatment. Using the real time monitoring system, dye laser (wavelength $590 \mathrm{~nm}$ ) photocoagulation was applied to the juxtafoveal lesion in her left eye (power, $250 \mathrm{~mW}$; size, 150-200 $\mu \mathrm{m}$; and duration, 0.2 second) (Fig 5). Her fixation point remained stable throughout the laser treatment as she fixated on the centre of the cross. The scale of the cross target was also useful for determining the distance between the edge of the $\mathrm{CNV}$ and the laser spot. In this case, her fixation point was approximately 100 $\mu \mathrm{m}$ from the edge of the photocoagulated juxtafoveal lesion. A fundus photograph taken immediately after photocoagulation shows a whitish laser burn close to the foveola (Fig 6).

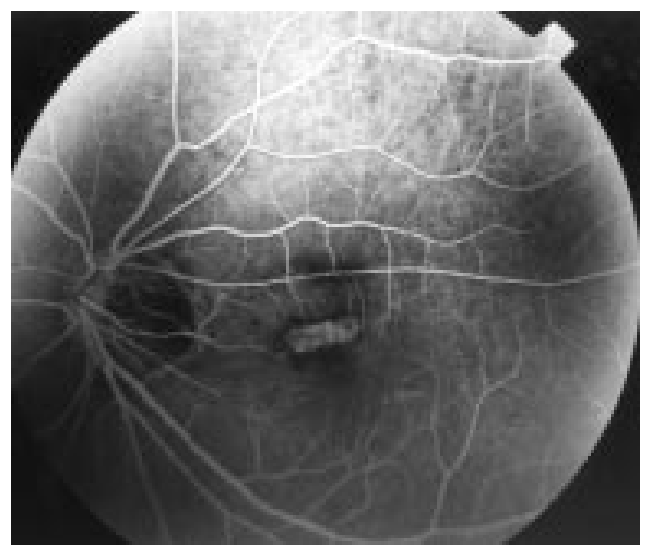

Figure 4 Fluorescein angiography reveals juxtafoveal choroidal neovascularisation in a 45 year old myopic female patient.

No damage to the foveola occurred because her corrected visual acuity improved to $20 / 20$ as a result of the resolution of the serous retinal detachment around the CNV 2 weeks after laser treatment. Ten months later her vision remained the same.

\section{Results}

Thirteen (48\%) of 27 patients answered that it was easier to keep the focus on the cross target image rather than on the aiming beam. The rest of the patients said that both were about equal. Fixation could be maintained throughout the procedure. The laser treatment was completed without foveal damage near the fixation point in all patients. The cross target image was very useful for the treatment of patients with foveolar subretinal haemorrhage because of disturbed foveal sensitivity.

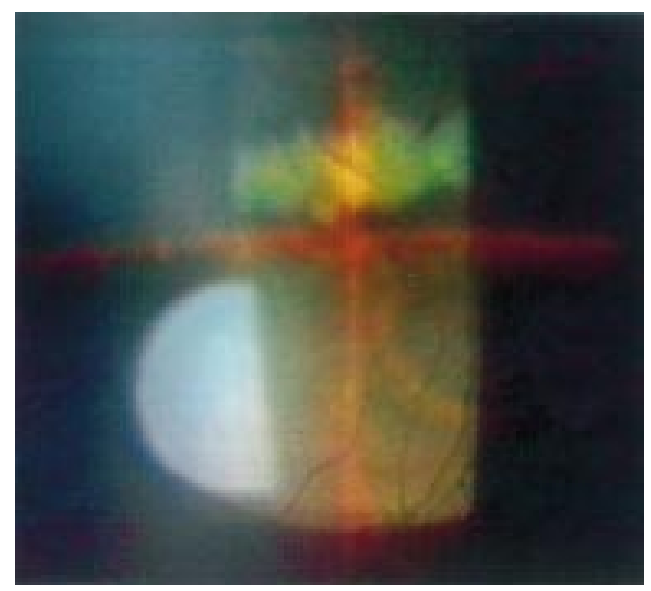

Figure 5 Photocoagulation treatment as seen through the slit lamp microscope and the contact lens. The scale on the target as seen in Figure 1 is not clearly defined because a print from an $S$-VHS videotape recording of the image is not as clear as the real image. The surgeon can read the scale on the real time image seen through the slit lamp microscope. The patient was asked to look at the centre of the cross target during laser treatment. The brightest white spots are reflections of the contact lens. The photocoagulated region is whitish at the end of the treatment. The fixation point remains the same at the centre of the cross target. (Slit lamp microscope magnification, $\times 20$.) Her fixation point was approximately $100 \mu \mathrm{m}$ from the edge of the

photocoagulated juxtafoveal lesion measured from the scale. 


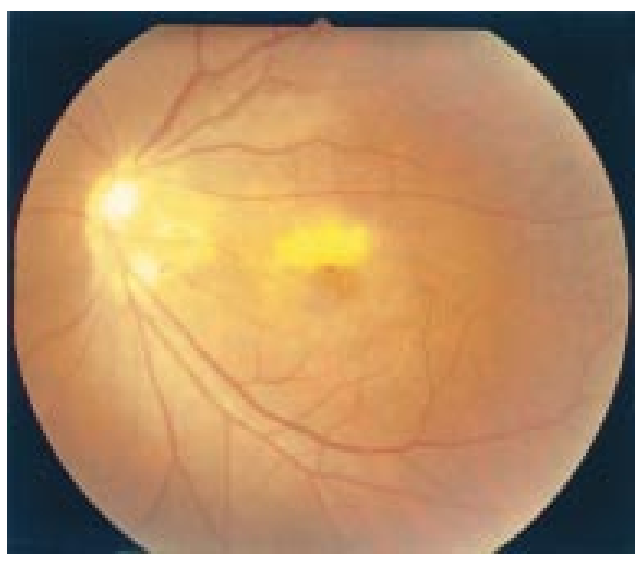

Figure 6 A fundus photograph taken immediately after photocoagulation shows whitish laser burn close to the foveola.

\section{Discussion}

A technique commonly used to determine the fixation point is by examining fundus photographs and fluorescein angiograms to locate the centre of the foveal avascular zone as the anatomical landmark. ${ }^{3}$ However, this is not foolproof in eyes in which the macula is affected, because there can be slight variation in fixation away from the centre of the foveal avascular zone. ${ }^{3}$ Scanning laser ophthalmoscopic microperimetry is a sensitive tool for detecting the fixation point. ${ }^{4}$ Although the results using microperimetry were helpful for determining the fixation point and the $\mathrm{CNV}$ margin, ${ }^{5}$ the location of the centre of fixation cannot be projected in real time onto the fundus during laser treatment.

At present, the only technique to detect the fixation point in real time is to ask the patient to look at the laser aiming beam. This method, however, is sometimes unstable and unreliable especially in patients with disturbed central vision. Moreover, at the time of aiming and photocoagulation, the aiming beam should be moved from the fixation point to the affected lesion to be treated.

In contrast, our novel system provides continuous monitoring of the fixation point during laser photocoagulation. Surgeons can determine the fixation point precisely not only by the anatomical landmark evaluated on fluorescein angiograms but also by real time monitoring of the functional fovea. Because $100 \mu \mathrm{m}$ corresponds to half of the scale of the cross in our system, surgeons can avoid accidental burns to the foveola even when treating lesions less than $100 \mu \mathrm{m}$ from the fixation points. We believe that laser photocoagulation to treat juxtafoveal lesions can be more accurately and safely performed using this system because of the patient's stable fixation and the continuous transmission of real time information to the surgeon about the fixation point.

1 Macular Photocoagulation Study Group. Laser photocoagulation for juxtafoveal choroidal neovascularization: fiveyear results from randomized clinical trials. Arch Ophthalmol 1994;112:500-9.

2 Macular Photocoagulation Study Group. The influence of treatment extent on the visual acuity of eyes treated with krypton laser for juxtafoveal choroidal neovascularization. Arch Ophthalmol 1995;113:190-4.

3 Zeffren BS, Applegate RA, Bradley A, et al. Retinal fixation point location in the foveal avascular zone. Invest Ophthalmol Vis Sci 1990;31:2099-105.

4 Timberlake GT, Mainster MA, Webb RH, et al. Retinal localization of scotomata by scanning laser ophthalmoscopy. Invest Ophthalmol Vis Sci 1982;22:91-7.

5 Rohrschneider K, Glück R, Becker M, et al. Scanning laser fundus perimetry before laser photocoagulation of well defined choroidal neovascularisation. $\mathrm{Br} \mathcal{f}$ Ophthalmol 1997;81:568-73.

6 Oshima Y, Harino S, Tano Y. Scanning laser ophthalmoscope microperimetric assessment in patients with successful laser treatment for juxtafoveal choroidal neovascularization. Retina 1998;18:109-17. 